\title{
Copper(I)-Mediated Asymmetric Boryl Substitution of Allyl Acetals
}

\section{Key words}

copper

boryl substitution

allyl acetals

General reaction:

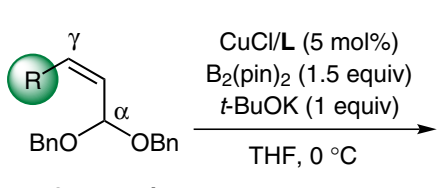

$0.5 \mathrm{mmol}$

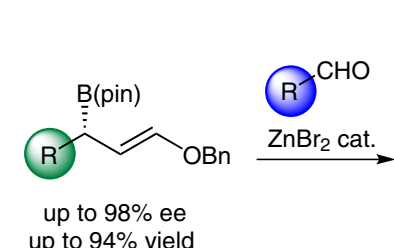
up to $94 \%$ yield

Selected examples (boryl substitution):

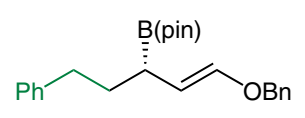

$94 \%$ yield $98 \%$ ee<smiles>CC(C)(C)OCCC(Br)C=COCc1ccccc1</smiles>

$93 \%$ yield $97 \%$ ee

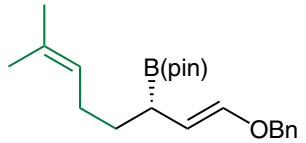
$91 \%$ yield $95 \%$ ee $\underline{B}$ (pin)<smiles>C[C](C)/C=C/OCc1ccccc1</smiles>

$88 \%$ yield

$96 \%$ ee<smiles>COCCC([18OH])/C=C/[OH2+]</smiles>
$81 \%$ yield
$97 \%$ ee<smiles>Cn1c(C(=O)OCC[C@H](Br)C=COc2ccccc2)cc2ccccc21</smiles>

3,3-Disubstituted cyclopentenes via a double boryl substitution/allylation strategy:<smiles>C1=CC2(CC1)OCCO2</smiles>

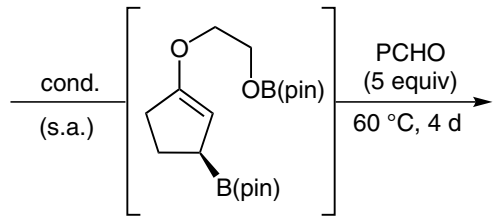<smiles>O[C@H](C1=CCCC1)c1ccccc1</smiles>
"B(pin)<smiles>C=C[C@@]1(C(C)C)CCCC1c1ccccc1</smiles>

$$
\begin{gathered}
\text { (2 equiv) } \\
\mathrm{PhMe}, 30^{\circ} \mathrm{C} \\
48 \mathrm{~h}
\end{gathered}
$$<smiles>OCCO[C@]1([C@@H](O)c2ccccc2)C=CCC1</smiles>

1. TBSCI, imidazole

2. cond. (s.a.)

L: Xantphos

$97 \%$ ee

$63 \%$ (2 steps)

$72 \%, 96 \%$ ee

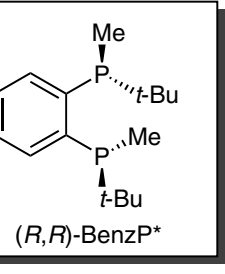

Significance: The authors describe a novel $\mathrm{Cu}$ mediated enantioselective and $\gamma$-selective boryl substitution of allyl acetals for the synthesis of enantioenriched $\alpha$-chiral linear or carbocyclic $(E)-(\gamma$ alkoxyallyl)boronates under mild conditions. The derived chiral boronates were used in aldehyde alIylation reactions rendering the respective 1,2-diol products in good yields and excellent enantioselectivity.

SYNFACTS Contributors: Mark Lautens, Steffen Kress Synfacts 2015, 11(2), 0155 Published online: 19.01.2015 DOI: 10.1055/s-0034-1379778; Reg-No.: L16314SF
Comment: Z-Substituted allyl acetates gave superior results over the analogous $E$-substrates. This versatile strategy was highlighted by an impressive example within the modular synthesis of 3,3-disubstituted cyclopentene. In this system the strategy was used twice, formally replacing both alkoxy groups of the acetal by two subsequent boryl substitution/aldehyde allylation reactions. Exceptional enantiocontrol and high yields were obtained. 